\title{
Comprehensive integrated analysis of gene expression datasets identifies key anti-cancer targets in different stages of breast cancer
}

\author{
MENG-TING GONG ${ }^{1}$, SHOU-DONG YE ${ }^{1}$, WEN-WEN LV², KAN HE${ }^{1}$ and WEN-XING LI ${ }^{3,4}$ \\ ${ }^{1}$ Center for Stem Cell and Translational Medicine, School of Life Sciences, Anhui University, Hefei, Anhui 230601; \\ ${ }^{2}$ Hongqiao International Institute of Medicine, Shanghai Tongren Hospital/Faculty of Public Health, Shanghai Jiao Tong \\ University School of Medicine, Shanghai 200025; ${ }^{3}$ State Key Laboratory of Genetic Resources and Evolution, Kunming \\ Institute of Zoology, Chinese Academy of Sciences, Kunming, Yunnan 650223; \\ ${ }^{4}$ Kunming College of Life Science, University of Chinese Academy of Sciences, \\ Kunming, Yunnan 650204, P.R. China
}

Received November 30, 2017; Accepted May 4, 2018

DOI: $10.3892 /$ etm.2018.6268

\begin{abstract}
Breast cancer is one of the primary threats to women's health worldwide. However, the molecular mechanisms underlying the development of breast cancer remain to be fully elucidated. The present study aimed to investigate specific target gene expression profiles in breast cancer tissues in general and in different breast cancer stages, as well as to explore their functions in tumor development. For integrated analysis, a total of 5 gene expression profiling datasets for 3 different stages of breast cancer (stages I-III) were downloaded from the Gene Expression Omnibus of the National Center for Biotechnology Information. Pre-processing of these datasets was performed using the Robust Multi-array Average algorithm and global renormalization was performed for all studies. Differentially expressed genes between breast cancer patients and controls were estimated using the empirical Bayes algorithm. The Database for Annotation, Visualization and Integrated Discovery web server was used for analyzing the enrichment of the differentially expressed genes in Gene Ontology terms of the category biological process and in Kyoto Encyclopedia of Genes and Genomes pathways. Furthermore, breast cancer target genes were downloaded from the Thomson Reuters
\end{abstract}

Correspondence to: Dr Kan He, Center for Stem Cell and Translational Medicine, School of Life Sciences, Anhui University, 111 Jiulong Road, Hefei, Anhui 230601, P.R. China

E-mail: hekan_803@163.com

Dr Wen-Xing Li, State Key Laboratory of Genetic Resources and Evolution, Kunming Institute of Zoology, Chinese Academy of Sciences, 32 Jiaochang Donglu, Kunming, Yunnan 650223, P.R. China

E-mail: liwenxing2016@gmail.com

Key words: breast cancer, gene expression, ribonucleotide reductase regulatory subunit $\mathrm{M} 2$, network, target genes
Integrity Database. We merged these target genes with the genes in breast cancer datasets. Analysis of anti-breast cancer gene networks was performed using the Genome-scale Integrated Analysis of Gene Networks in Tissues web server. The results demonstrated that the normal functions of the cell cycle, cell migration and cell adhesion were altered in all stages of breast cancer. Furthermore, 12 anti-breast cancer genes were identified to be dysregulated in at least one of the three stages. Among all of these genes, ribonucleotide reductase regulatory subunit M2 (RRM2) exhibited the highest degree of interaction with other interacting genes. Analysis of the network interactions revealed that the transcription factor of RRM2 is crucial for cancer development. Other genes, including mucin 1, progesterone receptor and cyclin-dependent kinase 5 regulatory subunit associated protein 3, also exhibited a high degree of interaction with the associated genes. In conclusion, several key anti-breast cancer genes identified in the present study are mainly associated with the regulation of the cell cycle, cell migration, cell adhesion and other cancer-associated cell functions, particularly RRM2.

\section{Introduction}

Breast cancer is one of the most common cancer types among women worldwide. According to global cancer statistics, an estimated $>1.6$ million patients were newly diagnosed and 500,000 breast cancer-associated mortalities occurred in 2012 worldwide (1). The Global Burden of Disease estimated that there were more than 1.7 million new cases and more than 545 thousand deaths in 2016 (2). In China, breast cancer alone is estimated to account for $15 \%$ of all newly diagnosed cancers in women, and its incidence has increased in the past decades (3). At present, chemotherapy is an important means of systemic therapy for breast cancer, in addition to surgical treatment. However, breast cancer may still be associated with poor prognosis, short survival time and rapid recurrence after therapy (4). The treatment and prognosis of breast cancer are affected by the expression levels of certain genes and proteins. For instance, triple-negative breast cancer 
[estrogen receptor (ER)-, progesterone receptor (PGR)- and human epidermal growth factor receptor 2 (HER2)-negative] is associated with poor prognosis and no targeted systemic therapy is currently available (5). However, patients with triple-positive breast cancer have a better prognosis and longer overall survival compared with triple-negative breast cancer patients (6).

The American Joint Committee on Cancer (AJCC) breast cancer staging system provides important information for the treatment and prognosis of this type of cancer (7). According to the 8th edition of the AJCC staging system, breast cancer may be divided into four main stages (stages I-IV) based on various factors, including the size of the tumor, the status of the lymph nodes and metastasis (7). Breast cancer staging is crucial for determining the extent of disease progression, as well as for containing and eliminating the cancer. The treatment of breast cancer depends partly on the stage of the disease, particularly in the case of targeted therapy. During breast cancer progression, diverse genetic signatures have been identified to drive processes of genome, transcriptome and epigenome remodeling (8-13). Therefore, it is necessary to select the most effective treatment options for breast cancer patients at different stages.

To date, numerous genes and pathways have been identified to be associated with breast cancer, and this information may be useful for studies into the pathological mechanisms and clinical treatment of breast cancer. According to the evidence provided by a functional study, the Wnt/ $\beta$-catenin signaling pathway controls cell fate in developmental processes and tumorigenesis, with $\beta$-catenin identified as a transforming factor (14). Based on a genome-wide assessment of allelic imbalances, an ATR/ATM-regulated DNA damage response network was identified to be activated in early human tumorigenesis, which may delay or prevent tumor progression (15). The results of a medical genomics study indicated that paired-box gene 2 may mediate endometrial carcinogenesis induced by tamoxifen, which has been widely used in the treatment of hormone-responsive breast cancer at all stages (16).

Thomson Reuters Integrity ${ }^{\mathrm{TM}}$ is a knowledge solution integrating biology, chemistry and pharmacology data (https://thomsonreutersintegrity.com). It contains exhaustive information on therapeutic drugs and gene targets for numerous complex human diseases. To the best of our knowledge, no previous study has compared the expression profiles of breast cancer target genes at different tumor stages. Gene expression profiling datasets at different stages of breast cancer are available from public databases, including the Gene Expression Omnibus of the National Center for Biotechnology Information (NCBI-GEO; http://www.ncbi. nlm.nih.gov/geo) $(17,18)$. The present study aimed to perform a comprehensive integrated analysis of gene expression datasets to identify key targets for breast cancer treatment and explore similarities and differences in the abnormalities of molecular signaling pathways/biological functions at different stages of breast cancer.

\section{Materials and methods}

Microarray data collection and pre-processing. Human breast cancer microarray datasets were searched and downloaded from the NCBI-GEO database in March 2016. The key words 'breast cancer', 'breast adenocarcinoma' and 'breast tumor' were used to perform a specific search. The selection criteria for the datasets were as follows: i) All datasets were genome-wide; ii) the samples of each dataset included breast cancer patients and controls; iii) the samples in tumor and control group were from breast tissue; iv) the dataset included different stages of breast cancer; and v) raw data were available. Datasets were excluded if: i) The number of samples was $<3$ for cases or controls; and ii) severe RNA degradation or an insufficient number of detected genes. Based on the aforementioned criteria, five datasets were finally selected for the integrated analysis [GSE10810 (19), GSE16391 (20), GSE29431 (21), GSE42568 (22) and GSE61304 (23)]. The integrated datasets included 257 breast cancer patients and 98 controls. A summary of the selected datasets is presented in Table I. All datasets had been generated using the Affymetrix Human Genome U133 Plus 2.0 Array. Among these five studies, one study included two stages of breast cancer (stages I-II), three studies included three stages of breast cancer (stages I-III), and one study included four stages of breast cancer (stages I-IV). As there was only 1 patient in stage IV, the datasets were divided into three subgroups (stage I-III).

R v3.2.2 (https://www.r-project.org/) was used for data pre-processing. The Robust Multichip Average (RMA) algorithm in the oligo-package was used to normalize the raw expression data and generate normalized gene expression intensity (24). Gene annotation, integration and re-normalization of the five datasets were performed using the custom-written Python code (25). Probes with no gene annotation or those that matched multiple gene symbols were removed. Next, the mean expression value of multiple probe IDs that matched an official gene symbol was calculated, and this value was considered to represent the expression intensity of the corresponding gene symbol. The re-normalization method was reported in a previous study (26).

Differential gene expression analysis. Differential gene expression analysis was performed using R v3.2.2 and the Bioconductor Library (http://www.bioconductor.org/). The empirical Bayes algorithm (function 'eBayes') in the limma package was used to detect differentially expressed genes between breast cancer and controls (27). Genes were considered to be upregulated if the logarithmic transformed fold-change $\log 2$ (FC) was $\geq \log 2(1.5)$ and the false discovery rate (FDR)-adjusted $\mathrm{P}$-value was $\leq 0.05$. Genes were considered to be downregulated if $\log 2(\mathrm{FC}) \leq-\log 2(1.5)$ and FDR-adjusted $\mathrm{P} \leq 0.05$. Differential expression analysis was performed for the whole cohort and the sub-groups (stage I-III). The control samples in the analysis for different stages were the same as the controls in the analysis for the whole cohort.

Enrichment analysis for differentially expressed genes in Gene Ontology $(\mathrm{GO})$ terms in the category biological process and in KEGG pathways. The Database for Annotation, Visualization and Integrated Discovery Bioinformatics Resources 6.7 was used to perform GO and KEGG pathway enrichment analysis (28). The input parameters were the list of differentially expressed genes. The significance level for enrichment was set at $\mathrm{P} \leq 0.05$. The 4 -set Venn diagram in InteractiVenn 
Table I. Summary of Gene Expression Omnibus breast cancer datasets used in the present study.

\begin{tabular}{llcc}
\hline Dataset ID & Author (year) & Samples (n) & Breast cancer stages \\
\hline GSE10810 & Pedraza (2010) & 58 & I, II, III \\
GSE16391 & Desmedt (2009) & $48^{\mathrm{a}}$ & I, II \\
GSE29431 & Lopez (2012) & 66 & I, II, III \\
GSE42568 & Clarke (2013) & 121 & I, II, III \\
GSE61304 & Aswad (2015) & 62 & I, II, III, IV \\
\hline
\end{tabular}

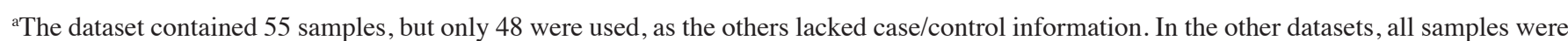
used.

Table II. Number of differentially expressed genes in breast cancer.

\begin{tabular}{lccrc}
\hline Group & Cases $^{\text {a }}$ & Mapped genes & Upregulated & Downregulated \\
\hline Entire cohort & 257 & 20307 & 153 & 183 \\
Stage I & 22 & 20307 & 53 & 275 \\
Stage II & 98 & 20307 & 167 & 309 \\
Stage III & 113 & 20307 & 202 & 165 \\
\hline
\end{tabular}

Values are expressed as $n .{ }^{a}$ With 98 controls in each group.

(http://www.interactivenn.net/) was used to present the GO terms in the category biological process in which the differentially expressed genes in the unstaged cohort and in different stages of breast cancer were enriched.

Breast cancer target gene analysis. Breast cancer-specific target genes were defined based on already available drugs or drugs under development that target these genes. All of these target genes were searched and downloaded from the Thomson Reuters Integrity Database. In total, a list of 344 breast cancer target genes were obtained, which were then mapped to the differentially expressed genes obtained in the present study for the whole cohort and for the sub-groups (stage I-III). The differentially expressed genes identified in the whole cohort and the sub-groups were overlapped with the 344 breast cancer target genes, and the 'barplot' function was used to present the results.

Gene interaction network analysis. A genome-scale integrated analysis of gene networks in breast cancer was performed using the Genome-scale Integrated Analysis of Gene Networks in Tissues (GIANT) web server (http://giant. princeton.edu/) (29). Based on the aforementioned results of the overlapping expression pattern of differentially expressed breast cancer target genes among the whole cohort and the sub-groups, the differentially expressed target genes in the unstaged cohort were used as input parameters to perform the gene network analysis. As the tissue options in the GIANT web server did not include breast tissue, 'all tissues' was selected to perform the analysis. The server generated a gene network of target genes and other genes that interacted with the target genes, and biological function enrichment analysis of the genes in the network was performed. The enriched biological processes were then presented using bar charts.

\section{Results and Discussion}

Overview of differentially expressed genes. The number of differentially expressed genes in breast cancer for each dataset is presented in Table II. A total of 153 upregulated and 183 downregulated genes were obtained for the whole cohort. In the unstaged cohort and in the stage I-II groups, more downregulated than upregulated genes were identified. However, in the stage-III group, the number of upregulated genes was higher than that of downregulated genes. The number of overlapping up- and downregulated genes among all four groups was 29 and 51, respectively.

GO and KEGG enrichment results. A Venn diagram displaying the enrichment results for the GO category biological process for the unstaged cohort and the individual stages is presented in Fig. 1. In the unstaged cohort, stage I-III groups, the differentially expressed genes were enriched in 138, 21, 119 and 136 GO terms, respectively. The top 10 enriched GO terms in the category biological process in each group are presented in Table III. Only one GO term in the category biological process, namely 'cell migration', was enriched in all four groups (the P-values for the unstaged cohort, stage I-III groups were $0.018,0.001,0.008$ and 0.035 , respectively). Activated cell migration is known to promote breast cancer progression (30). By contrast, inhibition of breast cancer cell migration contributes to successful treatment (31). Furthermore, 67 GO terms in the category biological process were enriched in the unstaged cohort, stage II-III groups, and 29 were enriched in the unstaged cohort and the stage III group. 


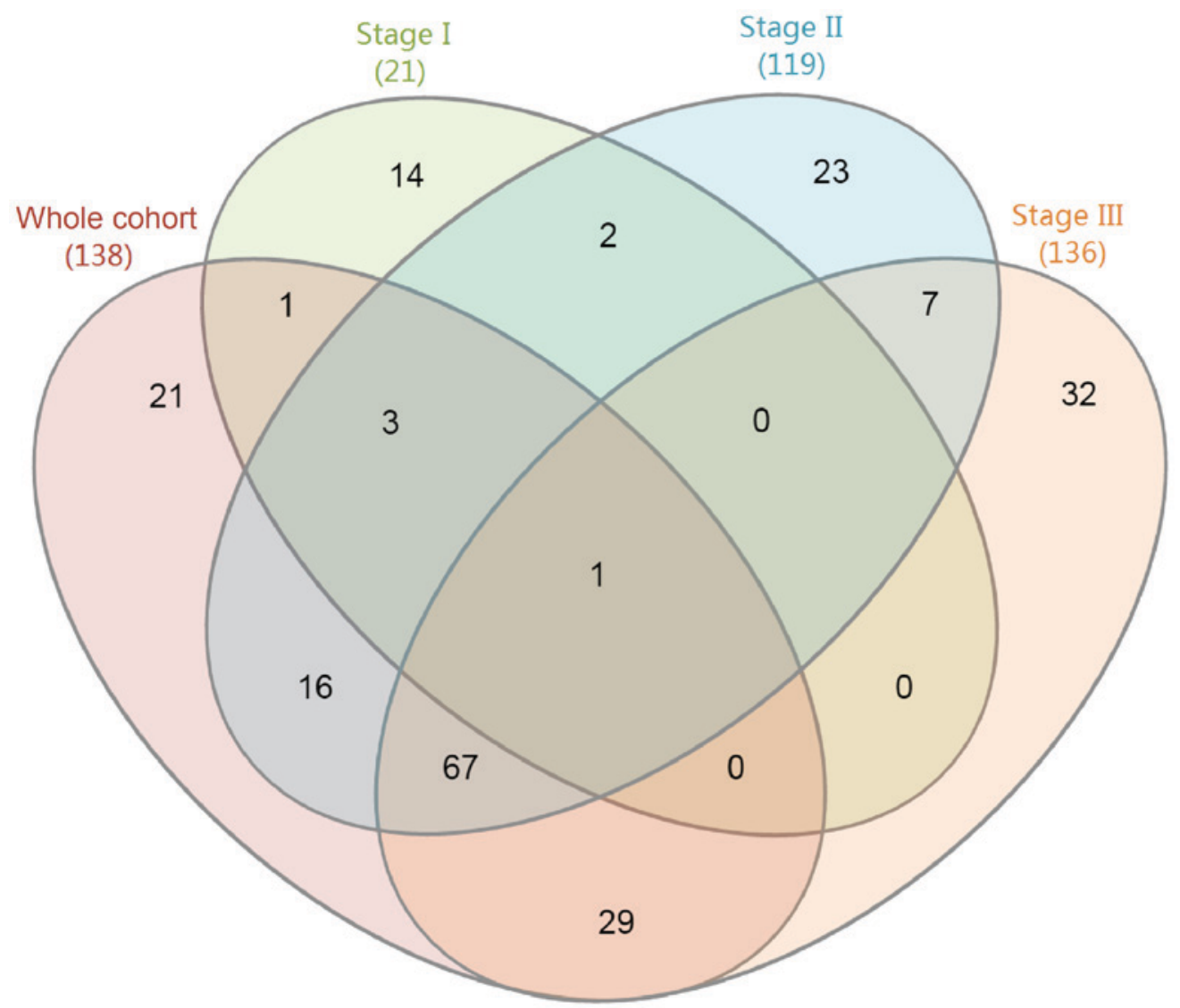

Figure 1. Venn Diagram of enriched Gene Ontology terms in the category Biological Process in breast cancer. The four groups (unstaged cohort, stage I-III) are represented by red, blue, cyan and orange color, respectively. The unstaged cohort contained 1 stage IV sample and 23 samples without stage information.

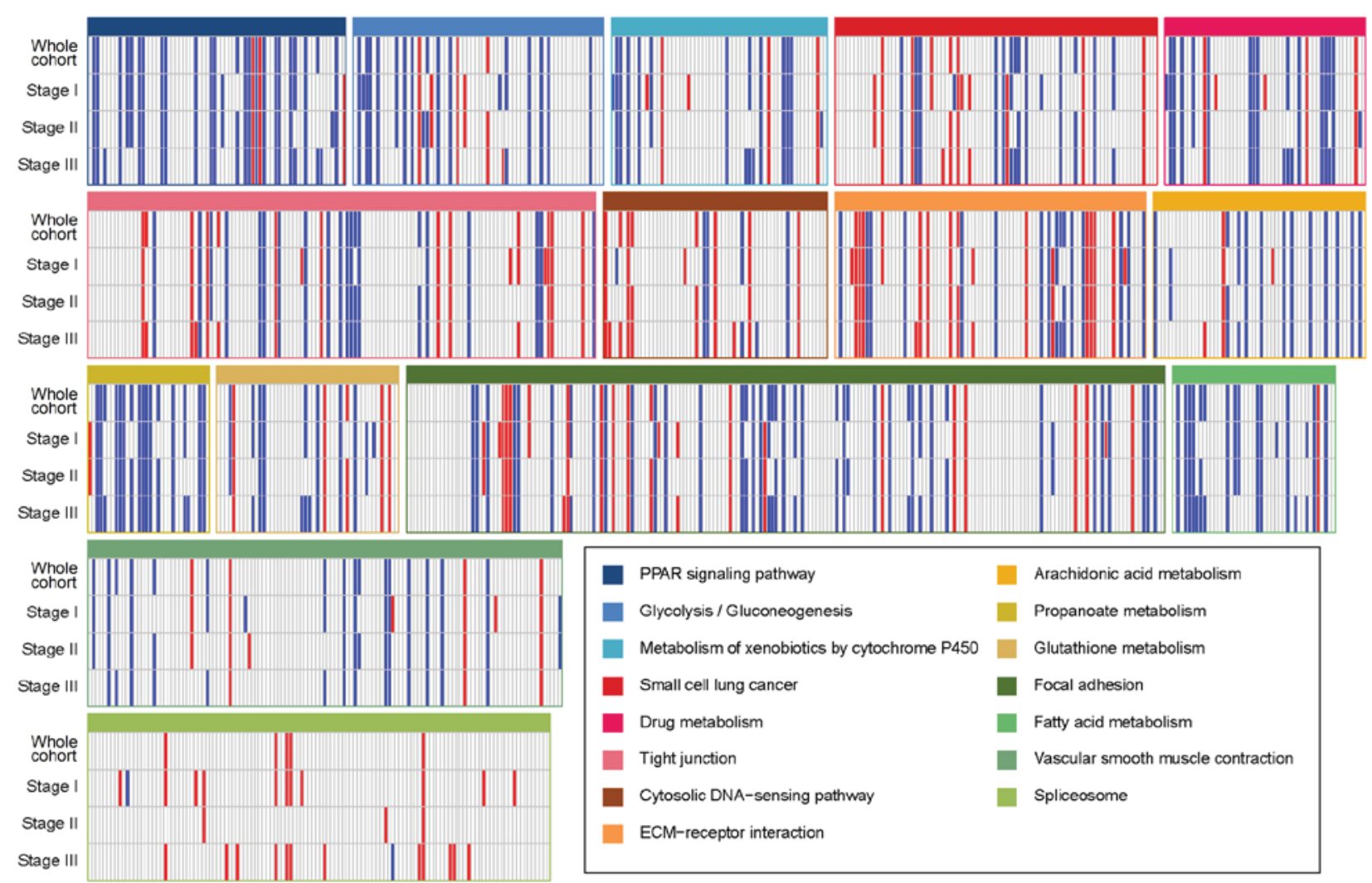

Figure 2. Gene expression profiles of enriched KEGG pathways in breast cancer. The 15 enriched KEGG pathways are represented by different colors. The red bars represent the upregulated genes and the blue bars represent the downregulated genes. KEGG, Kyoto Encyclopedia of Genes and Genomes; ECM, extracellular matrix; PPAR, peroxisome proliferator activated receptor. The unstaged cohort contained 1 stage IV sample and 23 samples without stage information. 

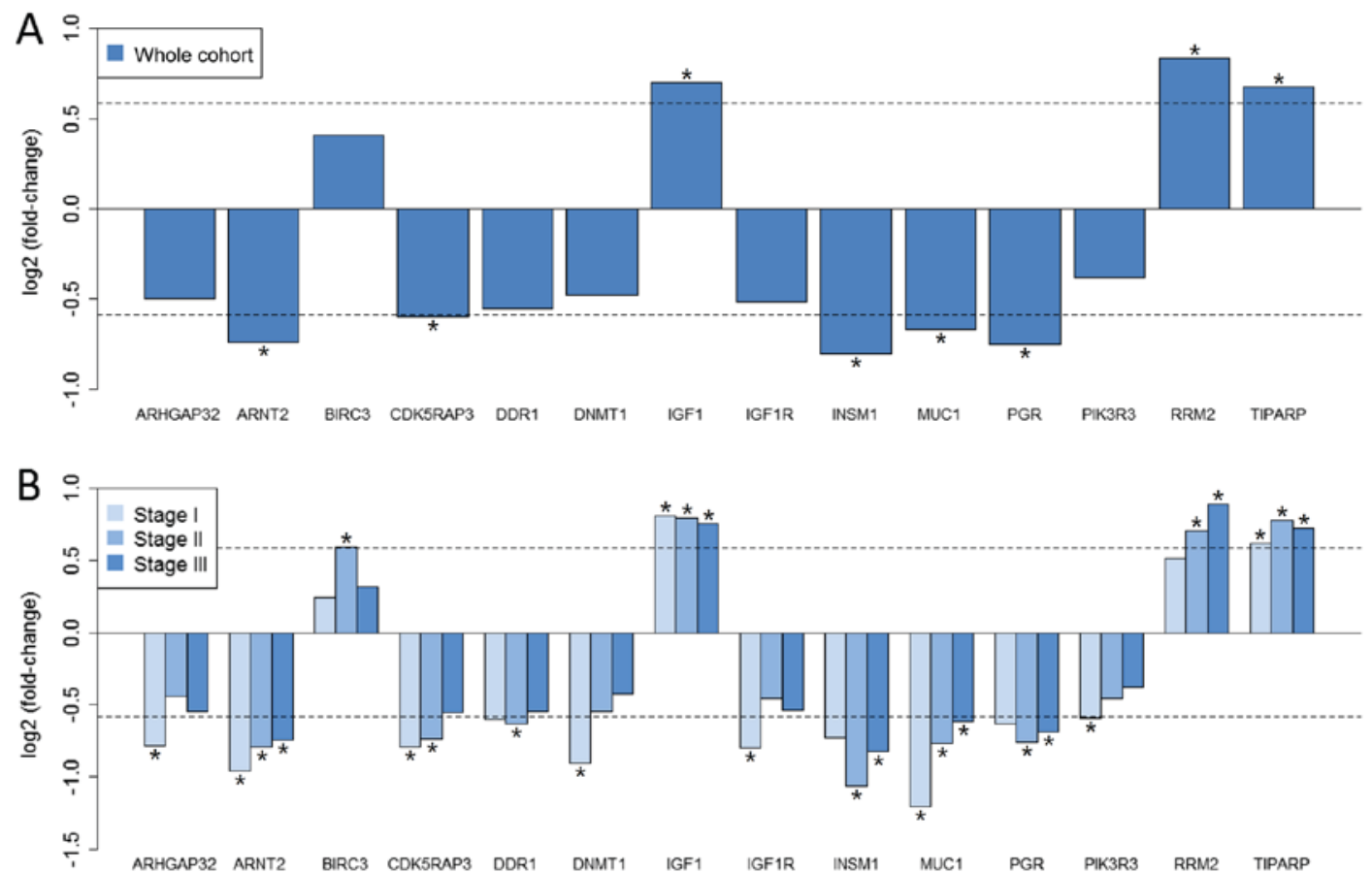

Figure 3. LogFC bar graph of mapped breast cancer-associated genes. (A) LogFC in the unstaged cohort. (B) LogFC in different stage groups. The horizontal dashed lines represent the $\log \mathrm{FC}$ cut-off for the up- and downregulated genes. "False discovery rate-adjusted $\mathrm{P}<0.05$. FC, fold change.

A

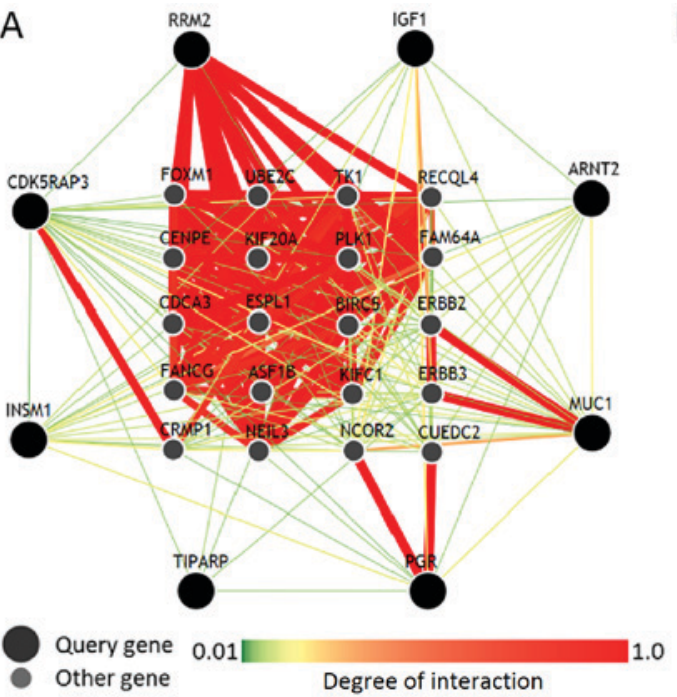

B

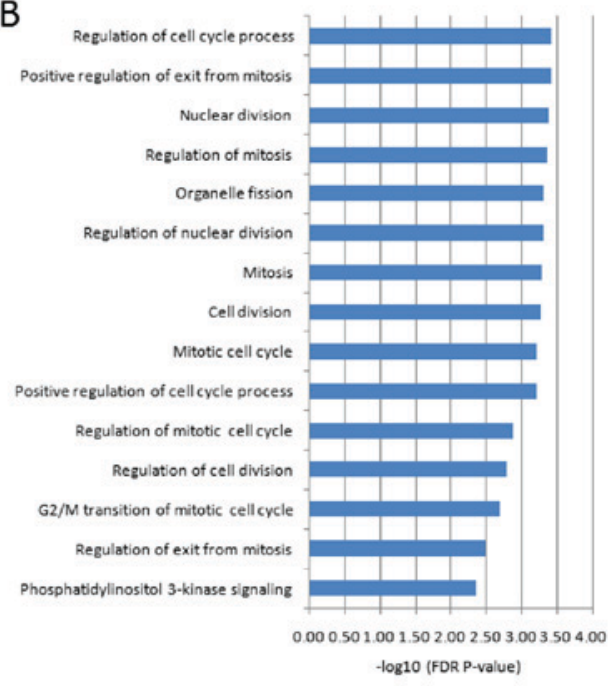

Figure 4. Genome-scale integrated analysis of gene networks in breast cancer. (A) The gene interaction network of breast cancer-associated target genes (query genes) and associated genes (other genes). (B) Top 15 Gene Ontology terms in the category biological process among the breast cancer-associated genes in the network. FDR, false discovery rate.

The KEGG pathways in which the differentially expressed genes of the four groups were enriched are presented in Table IV. The pathways 'glutathione metabolism', 'peroxisome proliferator activated receptor (PPAR) signaling pathway', 'metabolism of xenobiotics by cytochrome P450 (CYP)', 'arachidonic acid metabolism', 'drug metabolism' and 'tight junction' were significantly enriched in the whole cohort (P-values of $<0.001,0.001,0.004,0.018,0.025$ and 0.034 , respectively). Fig. 2 presents the gene expression profiles in the sets of enriched pathways in the whole cohort and the three sub-groups based on cancer stage. Overall, all of these pathways were severely affected. The pathways 'PPAR signaling', 'arachidonic acid metabolism', 'propanoate metabolism' and 'fatty acid metabolism' had a large number of downregulated genes across all groups. However, the 'spliceosome' pathway had more upregulated than downregulated genes. Glutamine has been reported to control cancer cell proliferation by activating signal transducer and activator of transcription 3 independent of glutamine metabolism (32). According to a study on mammary epithelial cell-specific PPAR $\gamma$ knockout mice, PPAR $\gamma$ expression and signaling has an inhibitory role in breast tumor progression (33). According to a previous study by our group, certain downstream genes mainly involved in lipid metabolism and adipocyte differentiation in the PPAR signaling pathway 
Table III. Top 10 enriched GO terms in the category biological process by the differentially expressed genes from the gene expression datasets for breast cancer.

\begin{tabular}{|c|c|}
\hline Group/GO term & P-value \\
\hline \multicolumn{2}{|l|}{ Entire cohort } \\
\hline Response to wounding & $<0.001$ \\
\hline Epithelial cell differentiation & $<0.001$ \\
\hline Response to endogenous stimulus & $<0.001$ \\
\hline Response to nutrient levels & $<0.001$ \\
\hline Epithelium development & $<0.001$ \\
\hline Regulation of hormone levels & $<0.001$ \\
\hline Defense response & $<0.001$ \\
\hline Response to drug & $<0.001$ \\
\hline Response to extracellular stimulus & $<0.001$ \\
\hline Response to steroid hormone stimulus & $<0.001$ \\
\hline \multicolumn{2}{|l|}{ Stage I } \\
\hline Cell migration & 0.001 \\
\hline Vasculature development & 0.002 \\
\hline Localization of cell & 0.004 \\
\hline Cell motility & 0.004 \\
\hline Endothelial cell migration & 0.005 \\
\hline Angiogenesis & 0.009 \\
\hline Odontogenesis & 0.010 \\
\hline Leukocyte migration & 0.012 \\
\hline Blood vessel development & 0.016 \\
\hline Blood vessel morphogenesis & 0.019 \\
\hline \multicolumn{2}{|l|}{ Stage II } \\
\hline Gland development & $<0.001$ \\
\hline Response to extracellular stimulus & $<0.001$ \\
\hline $\begin{array}{l}\text { Cellular di-, tri-valent inorganic } \\
\text { cation homeostasis }\end{array}$ & $<0.001$ \\
\hline Response to wounding & $<0.001$ \\
\hline $\begin{array}{l}\text { Di-, tri-valent inorganic cation } \\
\text { homeostasis }\end{array}$ & $<0.001$ \\
\hline Response to nutrient levels & $<0.001$ \\
\hline Response to nutrient & $<0.001$ \\
\hline Cellular cation homeostasis & $<0.001$ \\
\hline Cell-cell signaling & $<0.001$ \\
\hline Regulation of hormone levels & $<0.001$ \\
\hline \multicolumn{2}{|l|}{ Stage III } \\
\hline Response to endogenous stimulus & $<0.001$ \\
\hline Response to hormone stimulus & $<0.001$ \\
\hline $\begin{array}{l}\text { Response to steroid hormone } \\
\text { stimulus }\end{array}$ & $<0.001$ \\
\hline Response to organic substance & $<0.001$ \\
\hline Response to nutrient levels & $<0.001$ \\
\hline Response to wounding & $<0.001$ \\
\hline Oxidation reduction & $<0.001$ \\
\hline Epithelial cell differentiation & $<0.001$ \\
\hline Defense response & $<0.001$ \\
\hline Response to oxygen levels & $<0.001$ \\
\hline
\end{tabular}

Table IV. Enriched KEGG pathways by the differentially expressed genes from the gene expression datasets for breast cancer.

\begin{tabular}{|c|c|}
\hline Group/KEGG pathway & P-value \\
\hline \multicolumn{2}{|l|}{ Entire cohort } \\
\hline Glutathione metabolism & $<0.001$ \\
\hline PPAR signaling pathway & 0.001 \\
\hline Metabolism of xenobiotics by cytochrome P450 & 0.004 \\
\hline Arachidonic acid metabolism & 0.018 \\
\hline Drug metabolism & 0.025 \\
\hline Tight junction & 0.034 \\
\hline \multicolumn{2}{|l|}{ Stage I } \\
\hline Small cell lung cancer & 0.008 \\
\hline Focal adhesion & 0.009 \\
\hline ECM-receptor interaction & 0.035 \\
\hline Spliceosome & 0.038 \\
\hline Cytosolic DNA-sensing pathway & 0.047 \\
\hline \multicolumn{2}{|l|}{ Stage II } \\
\hline Tight junction & 0.034 \\
\hline Focal adhesion & 0.041 \\
\hline ECM-receptor interaction & 0.043 \\
\hline Vascular smooth muscle contraction & 0.043 \\
\hline Metabolism of xenobiotics by cytochrome P450 & 0.048 \\
\hline \multicolumn{2}{|l|}{ Stage III } \\
\hline Propanoate metabolism & 0.001 \\
\hline Glutathione metabolism & 0.001 \\
\hline PPAR signaling pathway & 0.001 \\
\hline Metabolism of xenobiotics by cytochrome P450 & 0.002 \\
\hline Fatty acid metabolism & 0.011 \\
\hline Arachidonic acid metabolism & 0.033 \\
\hline Glycolysis/gluconeogenesis & 0.041 \\
\hline Drug metabolism & 0.046 \\
\hline
\end{tabular}

KEGG, Kyoto Encyclopedia of Genes and Genomes; ECM, extracellular matrix; PPAR, peroxisome proliferator activated receptor.

were suppressed following downregulation of PPAR $\gamma$ in breast cancer, which may lead to tumorigenesis (13). It was previously reported that the expression of the CYP1A1, -2E1 and -3A4 was downregulated in tumor tissue, which may alter the biological effects of carcinogens and may represent a potential target for breast cancer chemoprevention (34). Previous studies demonstrated that induction of the expression of CYP2E1 reduces, whereas downregulation of CYP2E1 increases the migratory capacity, thereby promoting breast cancer cell progression (35). Thus, CYP2E1 may be associated with the regulation of breast cancer cell migration. CYP2E1 gene encodes a member of the cytochrome P450 superfamily of enzymes. Cytochrome P450 proteins are monooxygenases that catalyze many reactions involved in drug metabolism and synthesis of cholesterol, steroids and other lipids (36). It was previously demonstrated that differences in the expression of drug and xenobiotic metabolizing enzymes (DXME) markedly affect drug resistance. Substantial differences in DXME expression were identified in 
breast cancer patients of different ethnicities, which may affect pathways involved in drug metabolism (37).

Mapping of anti-breast cancer target genes. The differentially expressed breast cancer target genes were screened in the four groups. Subsequently, 8, 6, 9 and 7 breast cancer target genes from the differentially expressed genes in the unstaged cohort and stage I-III groups, respectively, were mapped. The combined set of these target genes contained 14 genes. Fig. 3 presents the $\log 2(\mathrm{FC})$ of these targets in each group. Insulin-like growth factor (IGF) 1 and TCDD-inducible poly (ADP-ribose) polymerase (TIPARP) were overexpressed in all groups, whereas aryl hydrocarbon receptor nuclear translocator 2 (ARNT2), INSM1, mucin (MUC)1 and PGR were downregulated in all groups, compared with the healthy controls. Overall, all these targets in the groups of different stages exhibited the same expression pattern as in the unstaged cohort. The IGF1 signaling axis has been reported to be crucial for tumorigenesis, and the activation of IGF1 receptor may promote breast cancer development by increasing glycolysis and promoting biomass production (38). Several polymorphisms of IGF1 pathway genes were reported to be associated with the risk of breast cancer (39). TIPARP is a poly(ADP-ribose) polymerase and a transcriptional repressor of the aryl hydrocarbon receptor, the polymorphism of which was previously reported to be associated with ovarian and breast cancer $(40,41)$. The mRNA expression level of ARNT2 was previously reported to be useful in determining the prognosis of breast cancer, and ARNT2 was reported to form functional complexes with hypoxia-inducible factor (HIF), which is a key to factor involved in tumor angiogenesis $(42,43)$. The results of small interfering RNA-mediated knockdown of ARNT2 suggested that ARNT2 may have a pivotal part in the modulation of HIF-1-regulated signaling and metabolism in MCF7 human breast cancer cells (43). The tumor oncoprotein MUC1 is a potential target in breast cancer immunotherapy, and the expression of MUC1 is absent or low in normal breast tissue, while it is high in breast cancer (44). PGR is one of the well-established breast cancer biomarkers, along with HER2/ERBB2 and ER (45).

Gene network of breast cancer targets. The present study identified 8 differentially expressed anti-breast cancer target genes in the whole cohort (Fig. 3), which were used to perform a genome-scale integrated analysis. The gene-gene interaction network and the top 15 enriched biological processes are shown in Fig. 4. Among all these targets, RRM2 displayed the highest degree of interaction with other interacting genes. Ribonucleotide reductase M2 (RRM2) is required for pyrimidine metabolism, and it is associated with aggressive tamoxifen-resistant breast tumors, whereas pharmacological inhibition and genetic knockdown of RRM2 sensitizes tumors to tamoxifen (46). In MCF-7 breast cancer cells, overexpression of RRM2 reduced the expression of ER $\alpha 66$ and caused an upregulation of the $36-\mathrm{kDa}$ variant of ER, ER $\alpha 36$, resulting in a reduction in the effectiveness of tamoxifen, which is widely used as an adjuvant therapy for patients with ER $\alpha$-positive tumors (47). Therefore, RRM2-associated metabolites may potentially be developed as prognostic markers for breast cancer. Furthermore, MUC1 exhibited a high degree of interaction with ERBB2 and ERBB3, PGR exhibited a high degree of interaction with nuclear receptor corepressor 2 and CUE domain containing 2, and cyclin-dependent kinase 5 regulatory subunit associated protein 3 exhibited a high degree of interaction with collapsin response mediator protein 1 (Fig. 4A). It has been demonstrated that the oncogenic MUC1 $\mathrm{C}$-terminal may act on the polycomb repressive complex 1 during epigenetic gene silencing, which is overexpressed in breast and other cancer types (48). The MUC1 oncoprotein was reported to be aberrantly overexpressed and associated with HER2/ERBB2 activation in breast cancer cells (49). The ERBB2/ERBB3 heterocomplex is a vital etiological feature of breast cancer, and it is important to understand its mechanisms of action to improve the design of novel, effective chemotherapeutics (50). As presented in Fig. 4B, the enriched biological processes of these target genes and interacting genes were mostly associated with cell cycle and mitosis. These results indicated that altered expression of these anti-breast cancer genes may severely affect the cell cycle and mitosis. Previous cell cycle-targeting agents have been reviewed, and emerging strategies for targeting mitosis in cancer have been refined and improved (51). Of note, in a recent study on functional mutagenesis screens in mice, human breast cancer susceptibility genes were, at large, not associated with cell cycle/mitosis genes (52). These results suggested that integration of human cancer transcriptomic data is required to identify breast cancer biomarkers with a high prognostic value. A limitation is that the tissue options in the GIANT web server did not include breast tissue, we choose 'all tissues' option to perform the analysis. This may have some influence on the result.

\section{Acknowledgements}

Not applicable.

\section{Funding}

This study was supported by the Natural Science Foundation Project of Anhui Province (grant no. 1508085QC63), the Scientific Research Foundation and Academic \& Technology Leaders Introduction Project (the 211 Project) of Anhui University (grant no. 10117700023) and the Student Research Training Program of Anhui University (grant nos. J10118520218 and J10118520307).

\section{Availability of data and materials}

The datasets used and/or analyzed during the current study are available from the corresponding author on reasonable request.

\section{Authors' contributions}

WXL and KH designed the present study. WXL, MTG and WWL performed breast cancer data collection. WXL, MTG and SDY conducted data analysis. WXL, KH and MTG wrote the manuscript. The final version of the manuscript has been read and approved by all authors, and each author believes that the manuscript represents honest work.

\section{Ethical approval and consent to participate}

Not applicable. 


\section{Patient consent for publication}

Not applicable.

\section{Competing interests}

The authors declare that they have no competing interests.

\section{References}

1. Torre LA, Bray F, Siegel RL, Ferlay J, Lortet-Tieulent J and Jemal A: Global cancer statistics, 2012. CA Cancer J Clin 65: 87-108, 2015

2. Global Burden of Disease Cancer Collaboration, Fitzmaurice C, Allen C, Barber RM, Barregard L, Bhutta ZA, Brenner H, Dicker DJ, Chimed-Orchir O, Dandona R, et al: Global, regional, and national cancer incidence, mortality, years of life lost, years lived with disability, and disability-adjusted life-years for 32 cancer groups, 1990 to 2015: A systematic analysis for the global burden of disease study. JAMA Oncol 3: 524-548, 2017.

3. Chen W, Zheng R, Baade PD, Zhang S, Zeng H, Bray F, Jemal A, Yu XQ and He J: Cancer statistics in China, 2015. CA Cancer J Clin 66: 115-132, 2016

4. O'Shaughnessy J: Extending survival with chemotherapy in metastatic breast cancer. Oncologist 10 (Suppl 3): S20-S29, 2005

5. Huang CS, Lin CH, Lu YS and Shen CY: Unique features of breast cancer in Asian women-breast cancer in Taiwan as an example. J Steroid Biochem Mol Biol 118: 300-303, 2010.

6. Reddy SM, Barcenas CH, Sinha AK, Hsu L, Moulder SL, Tripathy D, Hortobagyi GN and Valero V: Long-term survival outcomes of triple-receptor negative breast cancer survivors who are disease free at 5 years and relationship with low hormone receptor positivity. Br J Cancer 118: 17-23, 2018.

7. Giuliano AE, Connolly JL, Edge SB, Mittendorf EA, Rugo HS, Solin LJ, Weaver DL, Winchester DJ and Hortobagyi GN: Breast Cancer-Major changes in the American Joint Committee on Cancer eighth edition cancer staging manual. CA Cancer J Clin 67: 290-303, 2017.

8. Locke WJ and Clark SJ: Epigenome remodelling in breast cancer: Insights from an early in vitro model of carcinogenesis. Breast Cancer Res 14: 215, 2012.

9. Harvell DM, Kim J, O'Brien J, Tan AC, Borges VF, Schedin P, Jacobsen BM and Horwitz KB: Genomic signatures of pregnancy-associated breast cancer epithelia and stroma and their regulation by estrogens and progesterone. Horm Cancer 4 : $140-153,2013$

10. Mobasheri MB, Shirkoohi R, Zendehdel K, Jahanzad I, Talebi S, Afsharpad M and Modarressi MH: Transcriptome analysis of the cancer/testis genes, DAZ1, AURKC, and TEX101, in breast tumors and six breast cancer cell lines. Tumour Biol 36 : 8201-8206, 2015.

11. Nogales-Cadenas R, Cai Y, Lin JR, Zhang Q, Zhang W, Montagna $\mathrm{C}$ and Zhang ZD: MicroRNA expression and gene regulation drive breast cancer progression and metastasis in PyMT mice. Breast Cancer Res 18: 75, 2016.

12. Lang JE, Scott JH, Wolf DM, Novak P, Punj V, Magbanua MJ, Zhu W, Mineyev N, Haqq CM, Crothers JR, et al: Expression profiling of circulating tumor cells in metastatic breast cancer. Breast Cancer Res Treat 149: 121-131, 2015.

13. Li WX, He K, Tang L, Dai SX, Li GH, Lv WW, Guo YC, An SQ, Wu GY, Liu D, et al: Comprehensive tissue-specific gene set enrichment analysis and transcription factor analysis of breast cancer by integrating 14 gene expression datasets. Oncotarget 8: 6775-6786, 2016

14. Miyoshi K and Hennighausen L: Beta-catenin: A transforming actor on many stages. Breast Cancer Res 5: 63-68, 2003.

15. Bartkova J, Horejsí Z, Koed K, Krämer A, Tort F, Zieger K, Guldberg P, Sehested M, Nesland JM, Lukas C, et al: DNA damage response as a candidate anti-cancer barrier in early human tumorigenesis. Nature 434: 864-870, 2005.

16. Wu H, Chen Y, Liang J, Shi B, Wu G, Zhang Y, Wang D, Li R, Yi X, Zhang $\mathrm{H}$, et al: Hypomethylation-linked activation of PAX2 mediates tamoxifen-stimulated endometrial carcinogenesis. Nature 438: 981-987, 2005.

17. Edgar R, Domrachev M and Lash AE: Gene expression omnibus: NCBI gene expression and hybridization array data repository. Nucleic Acids Res 30: 207-210, 2002.
18. Barrett T, Wilhite SE, Ledoux P, Evangelista C, Kim IF, Tomashevsky M, Marshall KA, Phillippy KH, Sherman PM, Holko M, et al: NCBI GEO: Archive for functional genomics data sets-update. Nucleic Acids Res 41 (Database Issue): D991-D995, 2013.

19. Pedraza V, Gomez-Capilla JA, Escaramis G, Gomez C, Torné P, Rivera JM, Gil A, Araque P, Olea N, Estivill X and Fárez-Vidal ME: Gene expression signatures in breast cancer distinguish phenotype characteristics, histologic subtypes, and tumor invasiveness. Cancer 116: 486-496, 2010.

20. Desmedt C, Giobbie-Hurder A, Neven P, Paridaens R, Christiaens MR, Smeets A, Lallemand F, Haibe-Kains B, Viale G, Gelber RD, et al: The Gene expression Grade Index: A potential predictor of relapse for endocrine-treated breast cancer patients in the BIG 1-98 trial. BMC Med Genomics 2: 40, 2009.

21. Lopez FJ, Cuadros M, Cano C, Concha A and Blanco A: Biomedical application of fuzzy association rules for identifying breast cancer biomarkers. Med Biol Eng Comput 50: 981-990, 2012.

22. Clarke C, Madden SF, Doolan P, A herne ST, Joyce H, O'Driscoll L, Gallagher WM, Hennessy BT, Moriarty M, Crown J, et al: Correlating transcriptional networks to breast cancer survival: A large-scale coexpression analysis. Carcinogenesis 34: 2300-2308, 2013.

23. Aswad L, Yenamandra SP, Ow GS, Grinchuk O, Ivshina AV and Kuznetsov VA: Genome and transcriptome delineation of two major oncogenic pathways governing invasive ductal breast cancer development. Oncotarget 6: 36652-36674, 2015.

24. Carvalho BS and Irizarry RA: A framework for oligonucleotide microarray preprocessing. Bioinformatics 26: 2363-2367, 2010.

25. Li WX, Dai SX, Wang Q, Guo YC, Hong Y, Zheng JJ, Liu JQ, Liu D, Li GH and Huang JF: Integrated analysis of ischemic stroke datasets revealed sex and age difference in anti-stroke targets. PeerJ 4: e2470, 2016

26. Li WX, Dai SX, Liu JQ, Wang Q, Li GH and Huang JF: Integrated analysis of Alzheimer's disease and schizophrenia dataset revealed different expression pattern in learning and memory. J Alzheimers Dis 51: 417-425, 2016.

27. Ritchie ME, Phipson B, Wu D, Hu Y, Law CW, Shi W and Smyth GK: limma powers differential expression analyses for RNA-sequencing and microarray studies. Nucleic Acids Res 43: e47, 2015.

28. Huang da W, Sherman BT and Lempicki RA: Systematic and integrative analysis of large gene lists using DAVID bioinformatics resources. Nat Protoc 4: 44-57, 2009.

29. Greene CS, Krishnan A, Wong AK, Ricciotti E, Zelaya RA, Himmelstein DS, Zhang R, Hartmann BM, Zaslavsky E, Sealfon SC, et al: Understanding multicellular function and disease with human tissue-specific networks. Nat Genet 47: 569-576, 2015.

30. Liu Z, Zhan Y, Tu Y, Chen K, Liu Z and Wu C: PDZ and LIM domain protein 1(PDLIM1)/CLP36 promotes breast cancer cell migration, invasion and metastasis through interaction with a-actinin. Oncogene 34: 1300-1311, 2015.

31. Dong H, Claffey KP, Brocke S and Epstein PM: Inhibition of breast cancer cell migration by activation of cAMP signaling. Breast Cancer Res Treat 152: 17-28, 2015.

32. Cacace A, Sboarina M, Vazeille T and Sonveaux P: Glutamine activates STAT3 to control cancer cell proliferation independently of glutamine metabolism. Oncogene 36: 2074-2084, 2017.

33. Apostoli AJ, Roche JM, Schneider MM, SenGupta SK, Di Lena MA, Rubino RE, Peterson NT and Nicol CJ: Opposing roles for mammary epithelial-specific PPARg signaling and activation during breast tumour progression. Mol Cancer 14: 85, 2015.

34. El-Rayes BF, Ali S, Heilbrun LK, Lababidi S, Bouwman D, Visscher D and Philip PA: Cytochrome p450 and glutathione transferase expression in human breast cancer. Clin Cancer Res 9: 1705-1709, 2003.

35. Leung T, Rajendran R, Singh S, Garva R, Krstic-Demonacos M and Demonacos C: Cytochrome P450 2E1 (CYP2E1) regulates the response to oxidative stress and migration of breast cancer cells. Breast Cancer Res 15: R107, 2013.

36. Davydov DR, Davydova NY, Rodgers JT, Rushmore TH and Jones JP: Toward a systems approach to the human cytochrome P450 ensemble: Interactions between CYP2D6 and CYP2E1 and their functional consequences. Biochem J 474: 3523-3542, 2017.

37. Li Y, Steppi A, Zhou Y, Mao F, Miller PC, He MM, Zhao T, Sun Q and Zhang J: Tumoral expression of drug and xenobiotic metabolizing enzymes in breast cancer patients of different ethnicities with implications to personalized medicine. Sci Rep 7: 4747, 2017. 
38. Ter Braak B, Siezen CL, Lee JS, Rao P, Voorhoeve C, Ruppin E, van der Laan JW and van de Water B: Insulin-like growth factor 1 receptor activation promotes mammary gland tumor development by increasing glycolysis and promoting biomass production. Breast Cancer Res 19: 14, 2017.

39. Shi J,Aronson KJ, Grundy A, Kobayashi LC, Burstyn I, Schuetz JM, Lohrisch CA, SenGupta SK, Lai AS, Brooks-Wilson A, et al: Polymorphisms of insulin-like growth factor 1 pathway genes and breast cancer risk. Front Oncol 6: 136, 2016.

40. MacPherson L, Tamblyn L, Rajendra S, Bralha F, McPherson JP and Matthews J: 2,3,7,8-Tetrachlorodibenzo-p-dioxin poly(ADP-ribose) polymerase (TiPARP, ARTD14) is a mono-ADP-ribosyltransferase and repressor of aryl hydrocarbon receptor transactivation. Nucleic Acids Res 41: 1604-1621, 2013.

41. McKay J, Tenet V, Franceschi S, Chabrier A, Gheit T, Gaborieau V, Chopin S, Avogbe PH, Tommasino M, Ainouze M, et al: Immuno-related polymorphisms and cervical cancer risk: The IARC multicentric case-control study. PLoS One 12: e0177775, 2017.

42. Maltepe E, Keith B, Arsham AM, Brorson JR and Simon MC: The role of ARNT2 in tumor angiogenesis and the neural response to hypoxia. Biochem Biophys Res Commun 273: 231-238, 2000.

43. Qin XY, Wei F, Yoshinaga J, Yonemoto J, Tanokura M and Sone H: siRNA-mediated knockdown of aryl hydrocarbon receptor nuclear translocator 2 affects hypoxia-inducible factor-1 regulatory signaling and metabolism in human breast cancer cells. FEBS Lett 585: 3310-3315, 2011.

44. Yang E, Hu XF and Xing PX: Advances of MUC1 as a target for breast cancer immunotherapy. Histol Histopathol 22: 905-922, 2007.

45. Varga Z, Lebeau A, Bu H, Hartmann A, Penault-Llorca F, Guerini-Rocco E, Schraml P, Symmans F, Stoehr R, Teng X, et al: An international reproducibility study validating quantitative determination of ERBB2, ESR1, PGR, and MKI67 mRNA in breast cancer using MammaTyper ${ }^{\circledR}$. Breast Cancer Res 19: 55, 2017.
46. Putluri N, Maity S, Kommagani R, Creighton CJ, Putluri V, Chen F, Nanda S, Bhowmik SK, Terunuma A, Dorsey T, et al: Pathway-centric integrative analysis identifies RRM2 as a prognostic marker in breast cancer associated with poor survival and tamoxifen resistance. Neoplasia 16: 390-402, 2014.

47. Shah KN, Wilson EA, Malla R, Elford HL and Faridi JS: targeting ribonucleotide reductase $\mathrm{M} 2$ and $\mathrm{NF}-\kappa \mathrm{B}$ activation with didox to circumvent tamoxifen resistance in breast cancer. Mol Cancer Ther 14: 2411-2421, 2015.

48. Hiraki M, Maeda T, Bouillez A, Alam M, Tagde A, Hinohara K, Suzuki Y, Markert T, Miyo M, Komura K, et al: MUC1-C activates BMI1 in human cancer cells. Oncogene 36: 2791-2801, 2017.

49. Raina D, Uchida Y, Kharbanda A, Rajabi H, Panchamoorthy G, Jin C, Kharbanda S, Scaltriti M, Baselga J and Kufe D: Targeting the MUC1-C oncoprotein downregulates HER2 activation and abrogates trastuzumab resistance in breast cancer cells. Oncogene 33: 3422-3431, 2014.

50. Ishizawar RC, Miyake T and Parsons SJ: c-Src modulates ErbB2 and ErbB3 heterocomplex formation and function. Oncogene 26: 3503-3510, 2007.

51. Dominguez-Brauer C, Thu KL, Mason JM, Blaser H, Bray MR and Mak TW: Targeting mitosis in cancer: Emerging strategies. Mol Cell 60: 524-536, 2015.

52. Chen L, Jenjaroenpun P, Pillai AM, Ivshina AV, Ow GS, Efthimios M, Zhiqun T, Tan TZ, Lee SC, Rogers K, et al: Transposon insertional mutagenesis in mice identifies human breast cancer susceptibility genes and signatures for stratification. Proc Natl Acad Sci USA 114: E2215-E2224, 2017. International (CC BY-NC-ND 4.0) License. 